\title{
A Versatile General Approach to the Synthesis of Linearly Annelated $\pi$-Extended Porphyrins via 4,7-Dihydroisoindole Derivatives
}

\author{
Mikhail A. Filatov, Sergei E. Aleshchenkov, and Andrei V. Cheprakov ${ }^{\circledR}$ \\ Department of Chemistry, Moscow State University, Moscow, 119992, Russia \\ @Corresponding authorE-mail: avchep@elorg.chem.msu.ru
}

\begin{abstract}
A convenient and straightforward approach to the synthesis of linearly annelated extended porphyrins of tetrabenzoand tetranaphthoporphyrin series was developed. 4,7-Dihydroisoindole and its benzoannelated derivative, being the closest stable precursors of unstable $2 \mathrm{H}$-isoinsoles, are used in this method. Key features for this approach are its unprecedented synthetic versatility, tolerance for sensitive functional groups and the convenient introduction of meso-substituents. Aromatization of the annelated rings is conducted under mild conditions at the final stage of the synthesis. Tetraaryltetrabenzo- and tetranaphthoporphyrins are obtained using standard well-established procedures of porphyrin synthesis using common synthons.
\end{abstract}

Keywords: Extended porphyrins, tetrabenzoporphyrin, tetranaphthoporphyrin, tetraanthraporphyrin, dihydroisoindoles, Barton-Zard reaction.

\section{Introduction}

The so-called $\pi$-extended porphyrins, that is the subclass of porphyrins containing annelated aromatic or heteroaromatic systems, ${ }^{[1]}$ particularly those which bear acene-like linearly fused benzene rings (tetrabenzoporphyrins, tetranaphtho[2,3]porphyrins, tetraanthra[2,3]porphyrins), are of considerable interest both from fundamental and applied points of views. The electronic, optical and other properties of these porphyrinoids and their metal complexes attract attention in materials research, ${ }^{[2]}$ including the organic thin film transistors (OTFT) ${ }^{[3]}$ organic photovoltaic devices, ${ }^{[4]}$ biomedical imaging and sensing, ${ }^{[5]}$ photodynamic and boron neutron-capture therapy. ${ }^{[6]}$ Platinum complex of tetraphenyltetranaphthoporphyrin was recently shown to exhibit record efficiency in near-infrared OLED devices. ${ }^{[7]}$ In addition, the Pd and Pt complexes of LAEP have recently been demonstrated to be unique light harvesting materials for two-photon up-conversion based on triplet-triplet annihilation, allowing to transform low-intensity non-coherent red and near infrared light into shorter wavelength visible light, ${ }^{[8]}$ spawning applications for all-organic flexible displays ${ }^{[9]}$ and solar cell efficiency enhancement. ${ }^{[10]}$

A few synthetic approaches to such systems were developed, including the template condensation, ${ }^{[1,12,13]}$ Ono's method using the ethane-bridged tetrahydroisoindoles ${ }^{[14,15]}$ and the tetrahydroisoindole approach. ${ }^{[16,17]}$ Though quite useful in particular cases these methods share a common drawback, as at least one of the stages of each of these synthetic approaches rely on harsh conditions used either for enabling the condensation of rather unreactive substrates in template condensation, or for aromatization (demasking) of annelated rings in all other methods. As a consequence, tolerance to functional groups of all these methods is rather low, what substantially limits the synthetic scope.

The development of a more versatile approach towards linearly annelated extended porphyrins (LAEP) is therefore an important task. The main idea of such an approach should be to avoid any scope-restricting steps, which can be realized by moving the key synthons used in the synthetic procedures as close as possible to the formal progenitors of the LAEP class, which are $2 \mathrm{H}$-isoindoles, useless as such because of low stability. Such precursors are, as we have shown recently, 4,7-dihydroisoindole and its benzoannelated derivatives..$^{[18,19,20,21]}$ Though this approach might seem rather obvious, before our studies it has never been explicitly realized, most likely because of apparent doubts that such compounds and their derivatives along multi-step preparative chains used in porphyrin synthesis could survive the conditions used, particularly those involving acid and basecatalyzed reactions, which might have resulted in double bond shift and loss of properly positioned reaction centers. The first attempt to prepare 4,7-dihydroisoindole $\mathbf{1}^{[22]}$ indeed resulted in a failure and explicit statement of its poor stability and overall inapplicability towards the porphyrin chemistry. This instability, however, as we have shown ${ }^{[23]}$ was accounted for not by innate character of the compound, but rather by

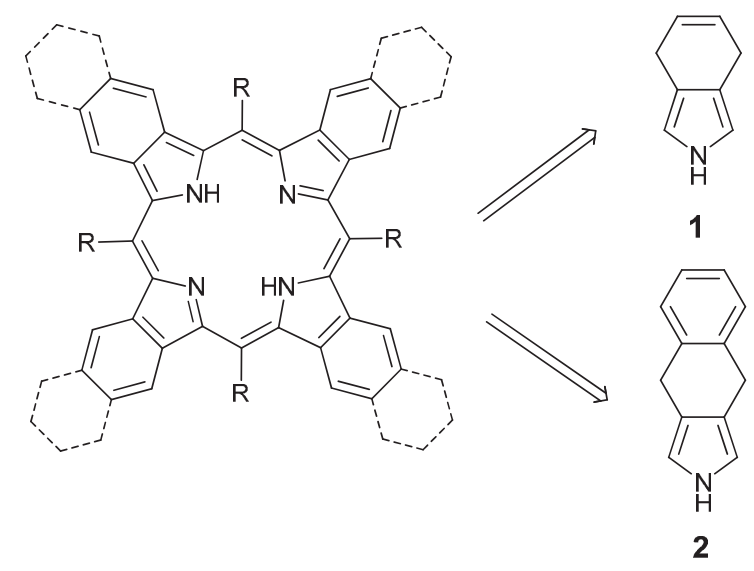

Scheme 1. Linearly annelated extended porphyrins and their synthetic precursors of 4,7-dihydroisoindole series. 
the way of synthesis used, and the same compound obtained using the Barton-Zard reaction turned out to be as stable as any other known 3,4-dialkylpyrrole.

In the present paper we summarize our achievements in the use of dihydroisoindole $\mathbf{1}$ and its benzoannelated analogue $\mathbf{2}$ in the synthesis of new tetrabenzo- and tetranaphthoporphyrins to emphasize the approach based on a common type of synthon, and common procedures involved to account for simplicity and versatility of the developed strategy.

\section{Experimental}

The reagents and solvents were purified according to standard procedures or used as received. Electronic absorption spectra were recorded on Perkin Elmer Lambda 40 spectrophotometer. MALDITOF mass-spectra were obtained with Brucker Daltonics Alphaflex II instrument without matrix (LDI mode) or using cinnamic acid as matrix (MALDI). NMR spectra were recorded using Brucker Avance-400 instrument (400 MHz for ${ }^{1} \mathrm{H}, 100 \mathrm{MHz}$ for $\left.{ }^{13} \mathrm{C}\right)$.

1-[(4-Methylphenyl)sulfonyl]-1,4-cyclohexadiene, 4. A mixture of ethynyl-p-tolylsulfone (5 g, 0.0278 ммоль) and $20 \mathrm{ml}$ 1,3-butadiene were stirred for $48 \mathrm{~h}$ in a thick-wall ampoule closed by heavy-duty teflone screw cap at room temperature. After opening the excess of butadiene was let out by spontaneous evaporation (hood!), and the solid residue was recrystallized from petroleum ether-ether (10:1) mixture to give colorless crystals (6.17 g, 95\%). M.p. $68-69^{\circ} \mathrm{C}$. Found: C 66.16, H $5.88 \% . \mathrm{C}_{13} \mathrm{H}_{14} \mathrm{O}_{2} \mathrm{~S}$ requires $\mathrm{C}$ 66.64, H 6.02. ${ }^{1} \mathrm{H}$ NMR $\left(\mathrm{CDCl}_{3}\right) \delta_{\mathrm{H}} \mathrm{ppm}: 7.75(\mathrm{~m}, 2 \mathrm{H}), 7.33(\mathrm{~m}$, $2 \mathrm{H}), 7.01(\mathrm{~m}, 1 \mathrm{H}), 5.72-5.60(\mathrm{~m}, 2 \mathrm{H}), 2.98-2.90(\mathrm{~m}, 2 \mathrm{H}), 2.85-$ $2.77(\mathrm{~m}, 2 \mathrm{H}), 2.43(\mathrm{~s}, 3 \mathrm{H}) .{ }^{13} \mathrm{C} \mathrm{NMR}\left(\mathrm{CDCl}_{3}\right) \delta_{\mathrm{C}} \mathrm{ppm}: 144.2,137.8$, 143.6, 129.8, 128.1, 122.8, 122.3, 27.0, 23.8, 21.6.

1-Alkoxycarbonyl-4,7-dihydroisoindoles, 5a,b. A solution of the respective isocyanoacetate $(0.25 \mathrm{~mol})$ in $20 \mathrm{ml}$ of THF (freshly distilled over $\mathrm{LiAlH}_{4}$ ) was added dropwise under argon to a solution of potassium tert-butylate $(3.06 \mathrm{~g}, 0.027 \mathrm{~mol})$ in $80 \mathrm{ml}$ of THF placed in a three-necked flask cooled by ice bath. Afterwards, a solution of sulfone $4(5.52 \mathrm{~g}, 0.024 \mathrm{~mol})$ in $20 \mathrm{ml}$ of THF was added dropwise to this mixture, upon addition the ice bath was removed and the mixture was stirred at room temperature for 6 $\mathrm{h}$. The reaction mixture was concentrated in vacuum to $c a .10 \mathrm{ml}$, diluted by $100 \mathrm{ml}$ of dichloromethane, washed by water and brine, dried by $\mathrm{Na}_{2} \mathrm{SO}_{4}$, filtered through a cake of silica, and evaporated in vacuum to dryness. Solid residues were recrystallized from petroleum ether.

5a, colorless crystals $(4.13 \mathrm{~g}, 80 \%)$. M.p. $110-111^{\circ} \mathrm{C}$. Found: C 71.11, H 8.04, N $6.20 \%$. $\mathrm{C}_{13} \mathrm{H}_{17} \mathrm{NO}_{2}$ requires C 71.21, H 7.81, N 6.39. ${ }^{1} \mathrm{H}$ NMR $\left(\mathrm{CDCl}_{3}\right) \delta_{\mathrm{H}}$ ppm: 9.59 (br. s, $\left.1 \mathrm{H}\right), 6.72(\mathrm{~d} J=2.40 \mathrm{~Hz}$, $1 \mathrm{H}), 5.94(\mathrm{~m}(\mathrm{AB}) J=10.4 \mathrm{~Hz}, 1 \mathrm{H}), 5.90(\mathrm{~m}(\mathrm{AB}) J=10.4 \mathrm{~Hz}, 1 \mathrm{H})$, $3.46(\mathrm{~m}, 2 \mathrm{H}), 3.25(\mathrm{~m}, 2 \mathrm{H}), 1.61(\mathrm{~s}, 9 \mathrm{H}) .{ }^{13} \mathrm{C} \mathrm{NMR}\left(\mathrm{CDCl}_{3}\right) \delta_{\mathrm{C}} \mathrm{ppm}$ : 161.6, 124.6, 124.0, 123.9, 118.8, 118.7, 118.2, 80.5, 28.6, 24.3, 22.5.

5b, white powder $(3.29 \mathrm{~g}, 73 \%)$. M. p. $87-88^{\circ} \mathrm{C}$. Found: C 68.77, H 7.00, N $7.03 \% . \mathrm{C}_{11} \mathrm{H}_{13} \mathrm{NO}_{2}$ requires C 69.09, H 6.85, N 7.32. ${ }^{1} \mathrm{H} \mathrm{NMR}\left(\mathrm{CDCl}_{3}\right) \delta_{\mathrm{H}}$ ppm: 8.97 (br. s., $\left.1 \mathrm{H}\right), 6.70(\mathrm{~m}, 1 \mathrm{H}), 5.87$ (m, 2H), 4.30 (q $J=7.12 \mathrm{~Hz}, 2 \mathrm{H}), 3.43(\mathrm{~m}, 2 \mathrm{H}), 3.22(\mathrm{~m}, 2 \mathrm{H}), 1.35$ $(\operatorname{tr} J=7.12 \mathrm{~Hz}, 3 \mathrm{H}) .{ }^{13} \mathrm{C} \mathrm{NMR}\left(\mathrm{CDCl}_{3}\right) \delta_{\mathrm{C}} \mathrm{ppm}: 161.6,124.9,124.4$, 123.8, 118.87, 117.5, 59.8, 24.0, 22.3, 14.5.

4,7-Dihydroisoindole, 1. Method A. A mixture of ester 5a (1 g, $4.56 \mathrm{mmol})$ and trifluoroacetic acid $(20 \mathrm{ml})$ was stirred for 30 min at room temperature under argon, upon which it was diluted by $50 \mathrm{ml}$ of dichloromethane, thoroughly washed by water, $10 \%$ solution of $\mathrm{NaHCO}_{3}$, brine, dried by $\mathrm{Na}_{2} \mathrm{SO}_{4}$, and evaporated in vacuum. The residue was passed through a short column of silica using dichloromethane as an eluent. Yellowish oil was obtained $(0.23 \mathrm{~g}, 55 \%)$. Method B. A mixture of ester $\mathbf{5 b}(1 \mathrm{~g}, 4.56 \mathrm{mmol})$, $\mathrm{KOH}(1.27 \mathrm{~g}, 0.023 \mathrm{mmol})$ and ethylene glycol $(30 \mathrm{ml})$ was re- fluxed for $30 \mathrm{~min}$. After cooling to room temperature, the mixture was diluted by dichloromethane $(50 \mathrm{ml})$, thoroughly washed by water ( 5 times by $200 \mathrm{ml}$ ) and brine, dried by $\mathrm{Na}_{2} \mathrm{SO}_{4}$, and evaporated in vacuum. The residue was passed through a short column of silica using dichloromethane as an eluent and an yellowish oil was obtained $(0.46 \mathrm{~g}, 85 \%)$. The product can be stored in a refrigerator at $-18^{\circ} \mathrm{C}$ for a few weeks, but preferably it is to be used for porphyrin synthesis soon after preparation. Purification to analytical quality was not pursued.

5,10,15,20-Tetraaryloctahydrotetrabenzoporphyrins, $\mathbf{6} \boldsymbol{a}, \boldsymbol{b}$. A mixture of dihydroisoindole $1(0.3 \mathrm{~g}, 2.5 \mathrm{mmol})$ and the corresponding benzaldehyde $(2.5 \mathrm{mmol})$ was dissolved in $250 \mathrm{ml}$ of dichloromethane (freshly distilled over $\mathrm{CaH}_{2}$ ). The mixture was stirred under argon for $10 \mathrm{~min}$, then boron trifluoride etherate $(0.071 \mathrm{~g}, 0.5 \mathrm{mmol})$ was added and the solution was stirred for $2 \mathrm{~h}$ at room temperature in a flask protected from light by aluminum foil, and then quenched by 2,3-dichloro-5,6-dicyanobenzoquinone ( $0.63 \mathrm{~g}, 2.8 \mathrm{mmol})$, optionally dissolved in $5 \mathrm{ml}$ of toluene. The mixture was stirred for $1 \mathrm{~h}$ at room temperature, washed by $10 \%$ aqueous solution of $\mathrm{Na}_{2} \mathrm{SO}_{3}$, brine, dried by $\mathrm{Na}_{2} \mathrm{SO}_{4}$, and evaporated in vacuum. The residue was purified by chromatography on silica using dichloromethane-acetic acid (50:1) mixture as an eluent, with further recrystallization from dichloromethane-MeOH.

6a, dark green powder $(0.13 \mathrm{~g}, 25 \%)$. M.p. $>300^{\circ} \mathrm{C} . \mathrm{m} / \mathrm{z}$ (MALDI-TOF) $823.39\left[(\mathrm{M}+\mathrm{H})^{+}\right]$, calculated for $\left(\mathrm{C}_{60} \mathrm{H}_{47} \mathrm{~N}_{4}+\mathrm{H}^{+}\right)$ 823.38. Free base. UV-vis $\left(\mathrm{CH}_{2} \mathrm{Cl}_{2}\right) \lambda_{\text {max }} \mathrm{nm}(\lg \varepsilon): 438$ (5.23), 532 (4.03), 568 (3.66), 576 (3.67), 614 (3.31). Dication salt. UV-vis (CH$\left.{ }_{2} \mathrm{Cl}_{2}, \mathrm{CF}_{3} \mathrm{COOH}\right) \lambda_{\max } \mathrm{nm}(\lg \varepsilon): 460$ (5.28), 610 (3.89), 670 (4.17). ${ }^{1} \mathrm{H}$ NMR $\left(\mathrm{CDCl}_{3}\right) \delta_{\mathrm{H}} \mathrm{ppm}: 8.41(\mathrm{~m}, 8 \mathrm{H}), 7.88(\mathrm{~m}, 12 \mathrm{H}), 5.48(\mathrm{~s}, 8 \mathrm{H})$, $3.20(\mathrm{~m}, 8 \mathrm{H}), 2.69(\mathrm{~m}, 8 \mathrm{H}), 1.50$ (br. s., $4 \mathrm{H}) .{ }^{13} \mathrm{C} \mathrm{NMR}\left(\mathrm{CDCl}_{3}\right) \delta_{\mathrm{C}}$ ppm: 143.4, 138.4, 136.4, 132.2, 130.0, 128.7, 123.0, 118.6, 25.6.

6b, dark green powder $(0.26$ g, $40 \%)$. M.p. $>300^{\circ} \mathrm{C} . \mathrm{m} / \mathrm{z}$ (MALDI-TOF) $1055.46\left[(\mathrm{M}+\mathrm{H})^{+}\right]$, calculated for $\left(\mathrm{C}_{68} \mathrm{H}_{55} \mathrm{~N}_{4} \mathrm{O}_{8}+\right.$ $\left.\mathrm{H}^{+}\right)$1055.40. Free base. UV-vis $\left(\mathrm{CH}_{2} \mathrm{Cl}_{2}\right) \lambda_{\max } \mathrm{nm}(\lg \varepsilon): 446$ (5.29), 540 (4.14), 574 (3.84), 616 (3.80), 682 (3.41). Dication salt. UVvis $\left(\mathrm{CH}_{2} \mathrm{Cl}_{2}, \mathrm{CF}_{3} \mathrm{COOH}\right) \lambda_{\text {max }} \mathrm{nm}(\lg \varepsilon): 468$ (5.34), 614 (4.05), 672 (4.27). ${ }^{2} \mathrm{H} \mathrm{NMR}\left(\mathrm{CDCl}_{3}\right) \delta_{\mathrm{H}}$ ppm: $8.57(\mathrm{~m}, 16 \mathrm{H}), 5.50(\mathrm{~m}, 8 \mathrm{H}), 4.15$ (s, $12 \mathrm{H}), 1.56$ (br. s., $4 \mathrm{H}) .{ }^{13} \mathrm{C} \mathrm{NMR}\left(\mathrm{CDCl}_{3}\right) \delta_{\mathrm{C}}$ ppm: 166.8, 143.0, 141.7, 136.3, 132.6, 131.5, 129.9, 122.8, 118.1, 52.7, 25.8.

5,10,15,20-Tetraaryltetrabenzoporphyrins, 7a,b. Porphyrin 6a or b $(0.05 \mathrm{~g})$ was dissolved in toluene $(10 \mathrm{ml})$. DDQ $(0.069 \mathrm{~g})$ was added and the solution was refluxed for $15 \mathrm{~min}$. On cooling to room temperature the mixture was diluted by dichloromethane, washed by $10 \%$ aq. solution of $\mathrm{Na}_{2} \mathrm{SO}_{3}$, brine, dried by $\mathrm{Na}_{2} \mathrm{SO}_{4}$, and evaporated in vacuum to dryness. The residue was recrystallized from dichloromethane-MeOH mixture. The obtained products were identical to materials described earlier. ${ }^{[21]}$

5,10,15,20-Tetraaryltetrabenzoporphyrins, 7c-e. Dihydroisoindole $1(0.23 \mathrm{~g}, 1 \mathrm{mmol})$ and the respective aldehyde $(1 \mathrm{mmol})$ were dissolved in $100 \mathrm{ml}$ of dichloromethane (freshly distilled over $\mathrm{CaH}_{2}$ ), stirred for $10 \mathrm{~min}$ in argon atmosphere. Boron trifluoride etherate $(0.028 \mathrm{~g}, 0.2 \mathrm{mmol})$ was added, and the mixture was stirred for $2 \mathrm{~h}$. On addition of DDQ $(0.17 \mathrm{~g}, 0.75 \mathrm{mmol})$ the mixture was stirred for $1 \mathrm{~h}$ at room temperature. Solvent was removed by evaporation in vacuum on a rotary evaporator, a solution of DDQ $(0.27 \mathrm{~g}, 1 \mathrm{mmol})$ in $30 \mathrm{ml}$ of toluene was added, and the mixture was refluxed for $30 \mathrm{~min}$, after what the solvent was evaporated in vacuum and the residue chromatographed on a short silica column using dichloromethane as an eluent. Emerald-green bands were collected, evaporated in vacuum and recrystallized from $\mathrm{CH}_{2} \mathrm{Cl}_{2}$ - Et $\mathrm{O}_{2}$ mixture.

7c, dark-green solid $\left(0.136\right.$ g, 48\%). M.p. $>300^{\circ} \mathrm{C} . \mathrm{m} / \mathrm{z}$ (MALDI-TOF) $1129.98\left[(\mathrm{M}+\mathrm{H})^{+}\right]$, calculated for $\left(\mathrm{C}_{60} \mathrm{H}_{34} \mathrm{Br}_{4} \mathrm{~N}_{4}+\mathrm{H}^{+}\right)$ 1129.95. Free base. UV-vis $\left(\mathrm{CH}_{2} \mathrm{Cl}_{2}\right) \lambda_{\text {max }} \mathrm{nm}(\lg \varepsilon): 464,558,596$, 636, 696. Dication salt. UV-vis $\left(\mathrm{CH}_{2} \mathrm{Cl}_{2}, \mathrm{CF}_{3} \mathrm{COOH}\right) \lambda_{\text {max }} \mathrm{nm}(\lg \varepsilon)$ : 498, 648, 698. ' $\mathrm{H}$ NMR $\left(\mathrm{CDCl}_{3}\right) \delta_{\mathrm{H}}$ ppm: 8.46 (AA'XX', 8H), 8.11 (AA'XX', 8H), 7.49 (AA'BB', 16H), 2.03 (br. s, 4H). ${ }^{13} \mathrm{C} \quad\left(\mathrm{CDCl}_{3}\right)$ 
$\delta_{\mathrm{C}}$ ppm: $140.9,137.7,137.3,133.0,130.9,129.7,125.9,124.3,113.7$.

7d, dark-green solid $\left(0.054\right.$ g, 23\%). M.p. $>300^{\circ} \mathrm{C} . \mathrm{m} / \mathrm{z}$ (MALDI-TOF) $935.44\left[(\mathrm{M}+\mathrm{H})^{+}\right]$, calculated for $\left(\mathrm{C}_{64} \mathrm{H}_{46} \mathrm{~N}_{4} \mathrm{O}_{4}+\right.$ $\left.\mathrm{H}^{+}\right)$935.36. Free base. UV-vis $\left(\mathrm{CH}_{2} \mathrm{Cl}_{2}\right) \lambda_{\text {max }} \mathrm{nm}(\lg \varepsilon): 466,540$, $586,634,696$. Dication salt. UV-vis $\left(\mathrm{CH}_{2} \mathrm{Cl}_{2}, \mathrm{CF}_{3} \mathrm{COOH}\right) \lambda_{\max } \mathrm{nm}$ (lge): 506, 650, 696. ' ${ }^{\mathrm{H}} \mathrm{NMR}\left(\mathrm{CDCl}_{3}\right) \delta_{\mathrm{H}} \mathrm{ppm}: 8.49$ (AA'XX', 8H), 7.53 (AA'BB', 8H), 7.46 (AA'XX', 8H), 7.45 (AA'BB', 8H), 4.18 (s, 12H), 1.77 (br. s., $4 \mathrm{H}) .{ }^{13} \mathrm{C} \mathrm{NMR}\left(\mathrm{CDCl}_{3}\right) \delta_{\mathrm{C}}$ ppm: 161.7, 141.6, 137.4, 132.5, 131.0, 129.2, 124.0, 115.2, 115.0, 114.2, 112.3, 55.7.

7e, dark-green solid $(0.035$ g, $15 \%)$. M.p. $>300^{\circ} \mathrm{C} . \mathrm{m} / \mathrm{z}$ (MALDI-TOF) $935.41\left[(\mathrm{M}+\mathrm{H})^{+}\right]$, calculated for $\left(\mathrm{C}_{64} \mathrm{H}_{46} \mathrm{~N}_{4} \mathrm{O}_{4}+\mathrm{H}^{+}\right)$ 935.36. Free base. UV-vis $\left(\mathrm{CH}_{2} \mathrm{Cl}_{2}\right) \lambda_{\text {max }} \mathrm{nm}(\lg \varepsilon): 460,546,588$, 636, 694. Dication salt. UV-vis $\left(\mathrm{CH}_{2} \mathrm{Cl}_{2}, \mathrm{CF}_{3} \mathrm{COOH}\right) \lambda_{\max } \mathrm{nm}(\lg \varepsilon)$ : 494, 640, 696. ${ }^{1} \mathrm{H} \mathrm{NMR}\left(\mathrm{CDCl}_{3}\right) \delta_{\mathrm{H}} \mathrm{ppm}: 8.30-8.46(\mathrm{~m}, 4 \mathrm{H}), 7.97$ $(\mathrm{m}, 4 \mathrm{H}), 7.65(\mathrm{~m}, 4 \mathrm{H}), 7.54(\mathrm{~m}, 8 \mathrm{H}), 7.44(\mathrm{~m}, 12 \mathrm{H}), 3.64$ (overlapped s., 12H), 1.30-1.70 (overlapped s., 4H).

3-Benzenesulfonyl-3,4-dihydronaphthalene, 9. A solution of $\mathrm{PhSCl}$ (7.34 g, $0.051 \mathrm{~mol})$ in $50 \mathrm{ml}$ of dichloromethane, prepared from $N$-chlorosuccinimide and thiophenol (stench!) according to published method, ${ }^{[26]}$ was added dropwise to a stirred solution of 1,4-dihydronaphthalene $(6.63 \mathrm{~g}, 0.051 \mathrm{~mol})$ in dry dichloromethane $(50 \mathrm{ml})$ at $0^{\circ} \mathrm{C}$ under argon. After addition, the precipitate of succinimide was filtered off. To the solution cooled to $0^{\circ} \mathrm{C} m$-chloroperbenzoic acid ( $22.8 \mathrm{~g}, 0.102 \mathrm{~mol})$ was slowly added portionwise, and the mixture was stirred for $12 \mathrm{~h}$ at room temperature. The precipitate was filtered off, and the solution was washed by aqueous $\mathrm{NaHCO}_{3}$, water, brine, dried by $\mathrm{Na}_{2} \mathrm{SO}_{4}$, and evaporated in vacuum to dryness. The solid residue was dissolved in $25 \mathrm{ml}$ of dichloromethane, and solution of DBU $(1.48 \mathrm{~g}, 0.016 \mathrm{~mol})$ in $5 \mathrm{ml}$ of dichloromethane was added. The mixture was stirred at room temperature for $1 \mathrm{~h}$, washed by water, brine, dried by $\mathrm{Na}_{2} \mathrm{SO}_{4}$, and evaporated in vacuum to dryness. The residue was recrystallized from ethanol and the white crystals were obtained $(11.02 \mathrm{~g}, 80 \%)$. M.p. $112^{\circ} \mathrm{C}$. Found: C 73.88, H $5.58 \%$. $\mathrm{C}_{16} \mathrm{H}_{14} \mathrm{O}_{2} \mathrm{~S}$ requires C 74.15, H 5.80. ${ }^{1} \mathrm{H}$ NMR $\left(\mathrm{CDCl}_{3}\right) \delta_{\mathrm{H}} \mathrm{ppm}$ : 7.66-7.72 (m, 2H), 7.35-7.40 (m, 1H), 7.21-7.28 (m, $2 \mathrm{H}), 7.02-7.06(\mathrm{~m}, 1 \mathrm{H}), 6.91-6.98(\mathrm{~m}, 2 \mathrm{H}), 6.70-6.76(\mathrm{~m}, 1 \mathrm{H}), 6.53-$ $6.58(\mathrm{~m}, 1 \mathrm{H}), 5.95-6.03(\mathrm{~m}, 1 \mathrm{H}), 3.96-4.03(\mathrm{~m}, 1 \mathrm{H}), 3.40-3.46(\mathrm{~m}$, $1 \mathrm{H}), 3.14-3.24(\mathrm{~m}, 1 \mathrm{H})$. Free base. UV-vis $\left(\mathrm{CH}_{2} \mathrm{Cl}_{2}\right) \lambda_{\max } \mathrm{nm}(\lg \varepsilon)$ : ${ }^{13} \mathrm{C} \mathrm{NMR}\left(\mathrm{CDCl}_{3}\right) \delta_{\mathrm{C}}$ ppm: 136.4, 133.7, 133.6, 131.5, 131.0, 129.5, 128.4, 128.2, 127.7, 127.0, 126.7, 118.9, 61.1, 28.0.

1-Ethoxycarbonyl-4,9-dihydrobenzo[f]isoindole, 10a. The compound was obtained according to the method described above for $\mathbf{5 b}$ in thefotm of yellowish solid (70\%). M.p. $155-156^{\circ} \mathrm{C}$. Found: C 74.67, $\mathrm{H}$ 6.27, N, 5.81. $\mathrm{C}_{15} \mathrm{H}_{15} \mathrm{NO}_{2}$ requires $\mathrm{C} 74.67, \mathrm{H} 6.27, \mathrm{~N} 5.81$. ${ }^{1} \mathrm{H}$ NMR $\left(\mathrm{CDCl}_{3}\right) \delta_{\mathrm{H}}$ ppm: 9.2 (br. s.,1H), $7.4(\mathrm{~m}, 1 \mathrm{H}), 7.3(\mathrm{~m}, 1 \mathrm{H})$, 7.28-7.20 (m, 2H), $6.86(\mathrm{~d} J=2.5 \mathrm{~Hz}, 1 \mathrm{H}), 4.42(\mathrm{q} J=7 \mathrm{~Hz}, 2 \mathrm{H})$, 4.23 (s, 2H), 3.95 (s, 2H), $1.46(\mathrm{t} J=7 \mathrm{~Hz}, 3 \mathrm{H}) .{ }^{13} \mathrm{C} \mathrm{NMR}\left(\mathrm{CDCl}_{3}\right)$ $\delta_{\mathrm{C}}$ ppm: 161.7, 135.3, 135.1, 129.4, 129.1, 126.4, 126.2, 126.1, 120.6, $118.3,117.6,60.2,28.4,27.1,14.8$.

5,10,15,20-Tetraaryltetranaphtho[2,3]porphyrins, $\mathbf{1 2 a} \boldsymbol{h}$. The porphyrins were obtained according to the method described above for porphyrins $7 \mathbf{c}-\mathbf{e}$.

12a, bluish-green shiny crystals (49\%). $\mathrm{m} / \mathrm{z}$ (MALDI-TOF) 1017.2 $\left[(\mathrm{M}+\mathrm{H})^{+}\right]$, calculated for $\mathrm{C}_{76} \mathrm{H}_{47} \mathrm{~N}_{4}$ 1015.4. Free base. UVvis (pyridine) $\lambda_{\max } \mathrm{nm}(\lg \varepsilon): 503$ (5.34), 680 (4.38), 731 (5.02). ${ }^{1} \mathrm{H}$ NMR (bis-trifluoroacetate, $\left.\mathrm{CDCl}_{3}\right) \delta_{\mathrm{H}} \mathrm{ppm}$ : 8.63-8.05 (m, 12H), $7.97(\mathrm{~s}+\mathrm{m}, 8+8 \mathrm{H}), 7.72(\mathrm{~m}, 8 \mathrm{H}), 7.50(\mathrm{~m}, 8 \mathrm{H}), 2.63$ (br.s., $4 \mathrm{H}) .{ }^{13} \mathrm{C}$ NMR (bis-trifluoroacetate, $\mathrm{CDCl}_{3}$ ) $\delta_{\mathrm{C}}$ ppm: 140.9, 139.7, 135.6, $132.9,130.6,130.2,129.9,129.5,127.5,125.4,112.6$.

12b, bluish-green crystals (45\%). $\mathrm{m} / \mathrm{z}$ (LDI-TOF) $1246.4\left[\mathrm{M}^{+}\right]$, calculated for $\mathrm{C}_{84} \mathrm{H}_{54} \mathrm{~N}_{4} \mathrm{O}_{8}$ 1246.4. Free base. UV-vis (pyridine) $\lambda_{\text {max }}$ nm (lge): 500 (5.32), 672 (4.42), 732 (5.05). Dication salt. UV-vis $\left(\mathrm{CH}_{2} \mathrm{Cl}_{2}, \mathrm{CF}_{3} \mathrm{COOH}\right) \lambda_{\text {max }} \mathrm{nm}$ (lge): 530 (5.27), 800 (4.88). ${ }^{1} \mathrm{H}$ NMR (bis-trifluoroacetate, $\mathrm{CDCl}_{3}$ ) $\delta_{\mathrm{H}}$ ppm: 8.80-8.64 (AA'BB', 16H), 7.94 (s, 8H), $7.73(\mathrm{~m}, 8 \mathrm{H}), 7.51(\mathrm{~m}, 8 \mathrm{H}), 4.16$ (s, 12H), 3.72 (br. s, $4 \mathrm{H}) .{ }^{13} \mathrm{C}$ (bis-trifluoroacetate, $\left.\mathrm{CDCl}_{3}\right) \delta_{\mathrm{C}} \mathrm{ppm}: 167.1,143.8,141.4$, $136.3,133.1,131.8,131.2,129.9,129.5,127.5,125.8,111.7,52.8 .{ }^{1} \mathrm{H}$
NMR (free base, 1,1,2,2-tetrachloroethane[D $\left.\left.\mathrm{D}_{2}\right], 363 \mathrm{~K}\right) \delta_{\mathrm{H}} \mathrm{ppm:} 8.66$ (AA'BB', 8H), 8.56 (AA'BB', 8H), 7.70 (s, 8H), 7.67 (m, 8H), 7.46 (m, 8H), 4.41 (s, 12H), 2.27 (br. S., 2H). ${ }^{13} \mathrm{C} \mathrm{NMR}$ (free base, $\mathrm{CDCl}_{3}$ pyridine[D $\mathrm{D}_{5}$ ], $\left.303 \mathrm{~K}\right) \delta_{\mathrm{C}}$ ppm: $167.3,148.5,146.5,134.7,131.1,130.6$, 130.4, 130.1, 129.0, 126.0, 125.5, 123.2, 113.4, 52.4

12c, bluish-green crystals (23\%). $\mathrm{m} / \mathrm{z}$ (LDI-TOF) 1194.7 $\left[\mathrm{M}^{+}\right]$, calculated for $\mathrm{C}_{76} \mathrm{H}_{42} \mathrm{~N}_{8} \mathrm{O}_{8}$ 1194.3. ${ }^{1} \mathrm{H}$ NMR (bis-trifluoroacetate, $\left.\mathrm{CDCl}_{3}\right) \delta_{\mathrm{H}} \mathrm{ppm}:$ 8.80-9.0 (AA'BB', 16H), $7.87(\mathrm{~s}, 8 \mathrm{H}), 7.71(\mathrm{~m}$, $8 \mathrm{H}), 7.57(\mathrm{~m}, 8 \mathrm{H}), 4.1$ (br. s., $4 \mathrm{H}) .{ }^{13} \mathrm{C}$ NMR (bis-trifluoroacetate, $\left.\mathrm{CDCl}_{3}\right) \delta_{\mathrm{C}}$ ppm: $149.2,144.5,140.8,136.9,133.1,129.6,129.3$, 128.6, 125.7, 125.3, 110.8. ${ }^{1} \mathrm{H}$ NMR (free base, $\mathrm{CDCl}_{3}$ ) $\delta_{\mathrm{H}} \mathrm{ppm}$ : 8.72 (AA'BB', 8H), 8.56 (AA'BB', 8H), 7.58 (br. s., 8H), 7.56 (br., $8 \mathrm{H}), 7.44$ ( br., $8 \mathrm{H}), 0.14$ (br. s., $2 \mathrm{H}$ ).

12d, dark-green crystals (34\%). ${ }^{1} \mathrm{HNMR}$ (bis-trifluoroacetate, $\left.\mathrm{CDCl}_{3}\right) \delta_{\mathrm{H}}$ ppm: 8.57 (AA'BB', 8H), $8.06(\mathrm{~s}, 8 \mathrm{H}), 7.78(\mathrm{~m}, 8 \mathrm{H}), 7.50$ (overlapped m., $8 \mathrm{H}+8 \mathrm{H}$ ), 4.22 (s, 12H), 3.42 (br. s., 4H). ${ }^{13} \mathrm{C} \mathrm{NMR}$ (bis-trifluoroacetate, $\mathrm{CDCl}_{3}$ ) $\delta_{\mathrm{C}}$ ppm: 158.9, 139.1, 134.8, 130.2, 127.4, 126.8, 124.3, 122.4, 112.6, 53.4. ${ }^{1} \mathrm{H}$ NMR (free base, $\mathrm{CDCl}_{3}$ ) $\delta_{\mathrm{H}}$ ppm: 8.34 (AA'BB' br. m., 8H), 7.77 (br. m., 8H), 7.50 (br. m., $8 \mathrm{H}+8 \mathrm{H}), 4.25$ (s, 12H), 1.56 (br. s., $4 \mathrm{H})$.

12e, dark-green crystals (27\%). m/z (LDI-TOF) $1330.4\left[\mathrm{M}^{+}\right]$, calculated for $\mathrm{C}_{76} \mathrm{H}_{42} \mathrm{Br}_{4} \mathrm{~N}_{4}$ 1330.0. ' $\mathrm{H}$ NMR (bis-trifluoroacetate, $\mathrm{CDCl}_{3}$ ) $\delta_{\mathrm{H}}$ ppm: 8.55 (AA'BB', 8H), 8.17 (AA'BB', 8H), 8.03 (s, $8 \mathrm{H}), 7.84(\mathrm{~m}, 8 \mathrm{H}), 7.60$ (m, 8H), 3.09 (br. s., $4 \mathrm{H}) .{ }^{13} \mathrm{C}$ (bis-trifluoroacetate, $\left.\mathrm{CDCl}_{3}\right) \delta_{\mathrm{C}} \mathrm{ppm}$ : 140.7, 138.1, 137.0, 133.3, 132.8, 129.4, 127.7, 125.4, 125.2, 111.2.

12f, dark-green crystals (17\%). m/z (LDI-TOF) 1254.7 [M+], calculated for $\mathrm{C}_{84} \mathrm{H}_{62} \mathrm{~N}_{4} \mathrm{O}_{8}$ 1254.5. ${ }^{1} \mathrm{H}$ NMR (bis-trifluoroacetate, $\left.\mathrm{CDCl}_{3}\right) \delta_{\mathrm{H}} \mathrm{ppm}: 8.26(\mathrm{~s}, 8 \mathrm{H}), 7.84(\mathrm{~m}, 8 \mathrm{H}), 7.80(\mathrm{~s}, 4 \mathrm{H}), 7.55(\mathrm{~m}$, $8 \mathrm{H}), 3.99(\mathrm{~s}, 24 \mathrm{H}) .{ }^{13} \mathrm{C}$ (bis-trifluoroacetate, $\mathrm{CDCl}_{3}$ ) $\delta_{\mathrm{C}} \mathrm{ppm}: 162.3$, 141.0, 133.2, 130.0, 129.6, 127.4, 125.7, 113.6, 103.2, 56.2.

12h, dark-green crystals (23\%). $\mathrm{m} / z$ (LDI-TOF) $1478.8\left[\mathrm{M}^{+}\right]$, calculated for $\mathrm{C}_{92} \mathrm{H}_{62} \mathrm{~N}_{4} \mathrm{O}_{16}$ 1478.4. ' $\mathrm{H}$ NMR (bis-trifluoroacetate, $\left.\mathrm{CDCl}_{3}\right) \delta_{\mathrm{H}} \mathrm{ppm}: 9.50(\mathrm{~s}, 8 \mathrm{H}), 9.39(\mathrm{~s}, 4 \mathrm{H}), 7.83(\mathrm{~s}, 8 \mathrm{H}), 7.67(\mathrm{~m}$, $8 \mathrm{H}), 7.49$ (m, 8H), 4.4 (s, 24H), 3.68 (br. s., $4 \mathrm{H}) .{ }^{13} \mathrm{C}$ (bis-trifluoroacetate, $\left.\mathrm{CDCl}_{3}\right) \delta_{\mathrm{C}}$ ppm: 168.2, 142.3, 141.6, 141.0, 134.5, 134.2, 133.9, 130.9, 130.5, 129.7, 127.1, 111.7, 54.9. ' $\mathrm{H}$ NMR (free base, $\left.\mathrm{CDCl}_{3}\right) \delta_{\text {н }}$ ppm: 9.55 (m, 4H), $9.42(\mathrm{~m}, 8 \mathrm{H}), 7.73$ (br. m., 8H), 7.57 (br. m., 8H), 4.10 (s, 12H), 1.67 (br. s., 2H).

Bis(3-ethoxycarbonyl-4,9-dihydrobenzo[f]isoindolyl)methane, 13. 1-Ethoxycarbonyl-4,7-dihydroisoindole 10a (2.41 g, 10 $\mathrm{mmol})$, dimethoxymethane $(0.38 \mathrm{~g}, 5 \mathrm{mmol})$ and p-toluenesulfonic acid hydrate $(0.095 \mathrm{~g}, 0.5 \mathrm{mmol})$ were dissolved in $50 \mathrm{ml}$ of acetic acid and the mixture was stirred for $12 \mathrm{~h}$ at room temperature. The mixture was poured in $200 \mathrm{ml}$ of water, the precipitate formed was filtered, dried in vacuum over $\mathrm{P}_{2} \mathrm{O}_{5}$ and recrystallized from ethanol. White powder $\left(2.35\right.$ g, 95\%). M.p. $155-156^{\circ} \mathrm{C}$. Found: C 74.92 , $\mathrm{H} 6.21, \mathrm{~N} 5.40 \% . \mathrm{C}_{31} \mathrm{H}_{30} \mathrm{~N}_{2} \mathrm{O}_{4}$ requires C 75.28, H 6.11, N 5.66. ${ }^{1} \mathrm{H}$ NMR $\left(\mathrm{CDCl}_{3}\right) \delta_{\mathrm{H}}$ ppm: 10.91 (br. s, $\left.2 \mathrm{H}\right), 7.17-7.26(\mathrm{~m}, 4 \mathrm{H}), 7.06-$ $7.14(\mathrm{~m}, 4 \mathrm{H}), 4.25(\mathrm{q}, 4 \mathrm{H}), 4.03(\mathrm{~s}, 4 \mathrm{H}), 3.89(\mathrm{~s}, 2 \mathrm{H}), 3.83(\mathrm{~s}, 4 \mathrm{H})$, $1.31(\mathrm{t}, 6 \mathrm{H}) .{ }^{13} \mathrm{C}\left(\mathrm{CDCl}_{3}\right) \delta_{\mathrm{C}} \mathrm{ppm}: 161.8,135.3,134.6,129.4,129.2$, 129.0, 125.9, 125.8, 116.2, 116.1, 59.5, 28.5, 26.5, 22.4, 14.7.

5,15-Bis(3,5-di-tert-butylphenyl)tetranaphtho[2,3]porphyrin, 14. Dipyrromethane 13 (1.48 g, 3 mmol), KOH (0.84 g, 15 $\mathrm{mmol}$ ), and $30 \mathrm{ml}$ of ethylene glycol were refluxed under argon for $1 \mathrm{~h}$. On cooling to room temperature the reaction mixture was diluted by $50 \mathrm{ml}$ of dichloromethane, thoroughly washed by water, brine, dried over $\mathrm{Na}_{2} \mathrm{SO}_{4}$ and evaporated in vacuum to afford brown solid, which was immediately used in porphyrin synthesis. This material $(0.5 \mathrm{~g}, 1 \mathrm{mmol})$ was dissolved in $60 \mathrm{ml}$ of dichloromethane. To this solution 3,5-di-tert-butylbenzaldehyde $(0.22$ $\mathrm{g}, 1 \mathrm{mmol})$ and trifluoroacetic acid $(0.015 \mathrm{~g}, 0.135 \mathrm{mmol})$ were added, and the mixture was stirred for $4 \mathrm{~h}$ under argon. DDQ $(0.34$ $\mathrm{g}, 1.5 \mathrm{mmol})$ in $20 \mathrm{ml}$ of toluene was added, and the mixture was stirred for $30 \mathrm{~min}$, after which it was evaporated in vacuum to dryness, the residue was dissolved in $30 \mathrm{ml}$ of toluene, DDQ $(0.45 \mathrm{~g}$, $2 \mathrm{mmol}$ ) was added, and the solution was refluxed for $30 \mathrm{~min}$ un- 
der argon. The mixture was evaporated in vacuum to dryness, the residue was chromatographed on silica using dichloromethane as eluent. Green fractions, after evaporation of the eluent in vacuum, were reprecipitated from dichloromethane by ether, the precipitate was thoroughly washed by ether. Dark-green powder $(0.21 \mathrm{~g}$, $38 \%$ ). Found: C 75.73, H 5.80, N, $3.77 \%$. Bis-trifluoroacetate ether solvate $\mathrm{C}_{84} \mathrm{H}_{72} \mathrm{~F}_{6} \mathrm{~N}_{4} \mathrm{O}_{4} \cdot \mathrm{Et}_{2} \mathrm{O}$ requires $\mathrm{C} 76.06, \mathrm{H}$ 5.95, N, 4.03. $\mathrm{m} / \mathrm{z}$ (LDI-TOF) $1086.29\left[\mathrm{M}^{+}\right]$, calculated for $\mathrm{C}_{92} \mathrm{H}_{62} \mathrm{~N}_{4} \mathrm{O}_{16}$ 1086.56. ${ }^{1} \mathrm{H}$ NMR (bis-trifluoroacetate, $\mathrm{CDCl}_{3}-\mathrm{CF}_{3} \mathrm{COOH}$ ) $\delta_{\mathrm{H}}$ ppm: 8.2-8.7 (br.s, 8H), 7.3-8.0 (br.s, 24H), 1.56 (s, 36H).

\section{Results and Discussion}

The Synthesis of 4,7-Dihydroisoindole and Tetrabenzoporphyrins

Dihydroisoindole 1 was prepared using a three-step synthesis based on commercially available tosylacetylene 3. ${ }^{[1]}$ This compound can as well be obtained in high yield from a cheaper commercial precursor bis-trimethylsilylacetylene. Tosylacetylene $\mathbf{3}$ is a moderately reactive dienophile, which reacts with butadiene-1,3 taken in excess as solvent in a thick-wall bomb, to afford the corresponding vinylic sulfone 4 transformed into 1-alkoxycarbonylderivatives of 4,7-dihydroisoindole 5a,b using the modified Barton-Zard reaction with the respective esters of isocyanoacetic acid. [2] 4,7-Dihydroisoindole 1 was then obtained either by solvolysis-decarboxylation of ester $\mathbf{5 a}$ in $\mathrm{CF}_{3} \mathrm{COOH}-\mathrm{CH}_{2} \mathrm{Cl}_{2}$ (1:1) mixture, or by saponification-decarboxylation of ester $\mathbf{5 b}$ in $\mathrm{KOH}$ solution in ethylene glycol at reflux, using the standard procedures widely applied in porphyrin chemistry without any modifications. In spite of easily predictable probability of the shift of the double bond from remote position to conjugation with aromatic pyrrolic ring, this complication did not ensue, as has been shown by careful analysis of reaction mixtures by NMR spectroscopy.

Dihydroisoindole 1 resembles in properties and stability other electron-rich pyrroles with alkyl substituents. It can be stored at $-20^{\circ} \mathrm{C}$ for several months, if carefully washed during the isolation to remove traces of acids. In this respect, the

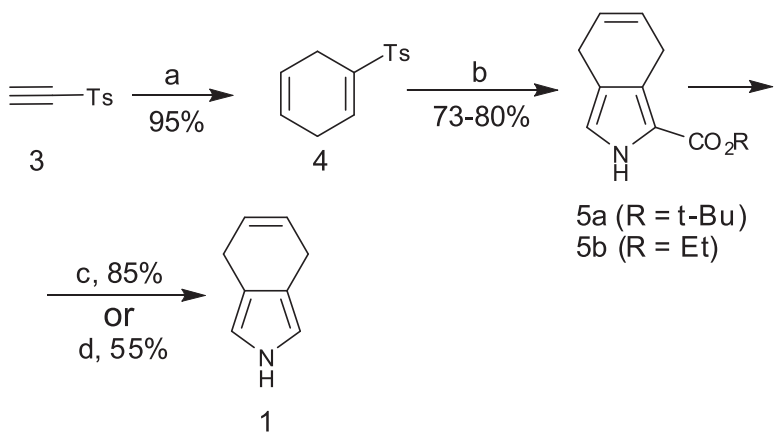

Scheme 2. a: 1,4-butadiene neat, $48 \mathrm{~h}$; b: $\mathrm{CNCH}_{2} \mathrm{CO}_{2} \mathrm{R},{ }^{\mathrm{t}} \mathrm{BuOK}$, THF, 4 h; c: $\mathrm{CF}_{3} \mathrm{COOH}-\mathrm{CH}_{2} \mathrm{Cl}_{2}, 1$ h; d: $\mathrm{KOH}, \mathrm{HOCH}_{2} \mathrm{CH}_{2} \mathrm{OH}$, $180^{\circ} \mathrm{C}, 30 \mathrm{~min}$.

alkaline deprotection procedure (step d in Scheme 2) furnishes more stable colorless material better surviving storage, while the acidic route (step c) is less time and labor consuming, but gives less stable dark-colored material to be preferred if it is to be used immediately. A further improvement in yield and purity can be achieved in the alkaline procedure, if the product is distilled off along with ethylene glycol during the reaction. Similarity between pyrrole 1 and other 3,4-dialkylpyrroles, particularly 4,5,6,7-tetrahydroisoindole enables to use welldeveloped procedures for the assembly of porphyrins using all such synthons.

As a first application of dihydroisoindole $\mathbf{1}$ we have developed the synthesis of tetraaryltetrabenzoporphyrins. A standard condensation with aldehydes under Lindsey's conditions was used, and the reaction mixtures after condensation were quenched with DDQ to afford octahydrotetrabenzoporphyrins 6 in good yields.
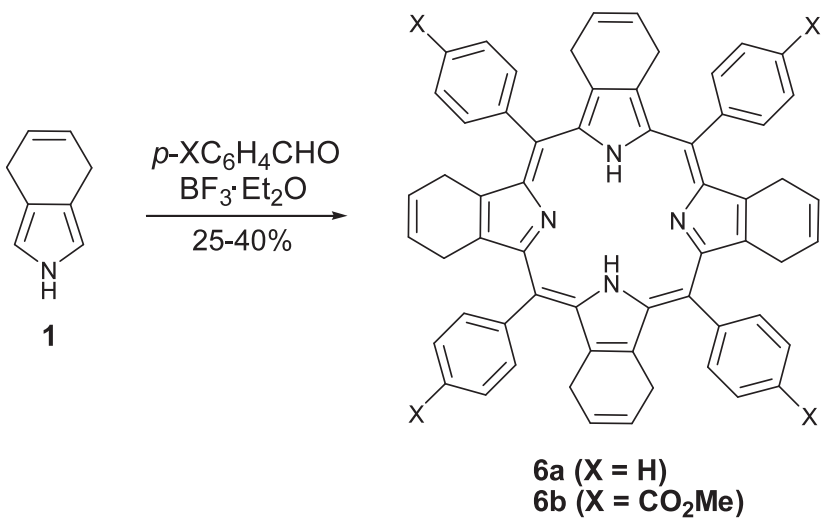

The porphyrins 6 were reliably characterized by ${ }^{1} \mathrm{H}$ and ${ }^{13} \mathrm{C}$ NMR spectroscopy, and MALDI TOF mass-spectrometry to contain isolated double bonds. Interestingly, neither double bond shifts nor aromatization of annelated rings took place, though both these sophistications were expectable. In a separate study we show that these porphyrins can be used to access new extended porphyrins using the addition reactions at the double bonds. ${ }^{[1]}$ Nevertheless, a full aromatization of all annealated rings can be performed very simply by the addition of extra amount of DDQ and mild heating. At $100^{\circ} \mathrm{C}$ the full conversion of porphyrins 6 to the respective tetrabenzoporphyrins was quantitative after 5-10 min.

The process can be performed in a one-pot manner without the isolation of intermediate porphyrins $\mathbf{6}$. After quenching of porphyrinogene, dichloromethane is substituted for toluene, 4 equivalents of DDQ are added and the mixture is heated for $10 \mathrm{~min}$ to afford tetrabenzoporphyrins 7 in the good yields. The purity of the products is high enough to allow for exclusion of chromatography in most cases, and pure materials are obtained by filtration through silica cake and recrystallization.

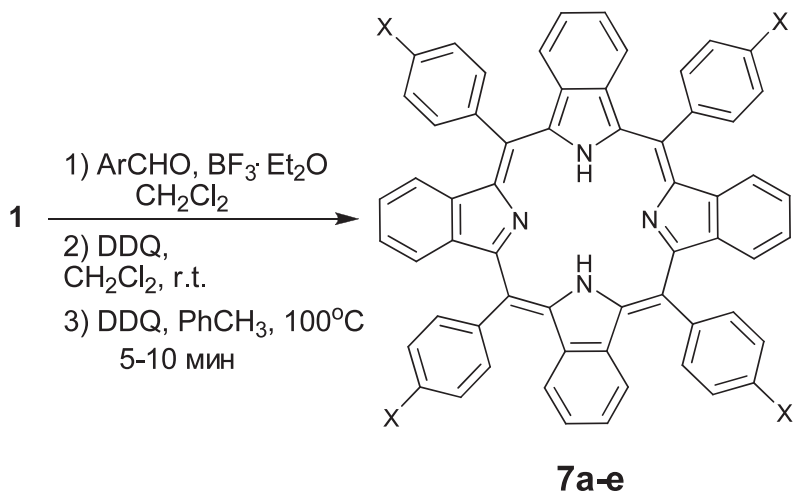

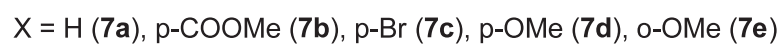


The developed procedure is in fact the first direct synthesis of tetrabenzoporphyrins performed under standard conditions used for the synthesis of regular porphyrins. Both donor and electron-withdrawing substituents are well tolerated.

\section{The Synthesis of 4,9-Dihydrobenzo[f]isoindole and Tetranaphthoporphyrins}

The extension of dihydroisoindole method to tetranaphthoporphyrins requires benzo-annelated dihydroisoindole $\mathbf{8}$. This compound was obtained by us earlier ${ }^{[18]}$ starting from naphthalene, which is readily reduced to 1,4-dihydronaphthalene by sodium in alcohol. ${ }^{[1]}$ Further transformations involve the transformation of this olefin into sulfone 9 according to the Hopkins-Fuchs method ${ }^{[2]}$ and further to tert-buthoxycarbonyl derivative of 4,9-dihydrobenzo[f]isoindole $\mathbf{1 0}$.
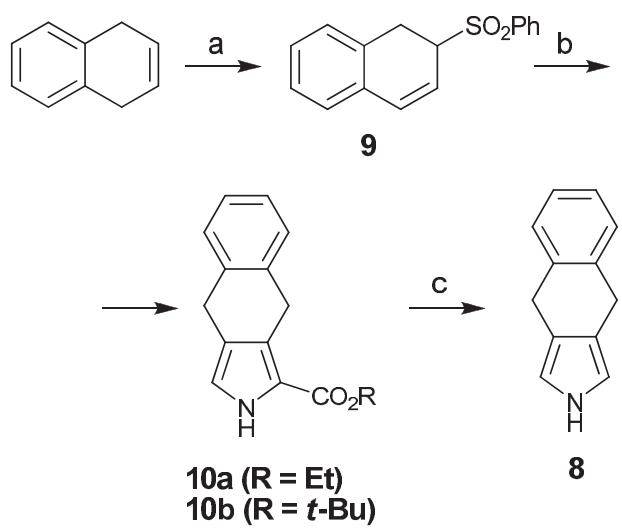

Scheme 3. a: 1 . $\mathrm{PhSCl}, \mathrm{CH}_{2} \mathrm{Cl}_{2}, 20^{\circ} \mathrm{C}, 12 \mathrm{~h}$; 2 . oxone, $\mathrm{MeOH}-$ $\mathrm{H}_{2} \mathrm{O}, 20^{\circ} \mathrm{C}, 12 \mathrm{~h} ; 3 . \mathrm{DBU}, \mathrm{CH}_{2} \mathrm{Cl}_{2}, 20^{\circ} \mathrm{C}, 30 \mathrm{~min}$; b: $\mathrm{CNCH}_{2} \mathrm{CO}_{2} \mathrm{R}$, ${ }^{t} \mathrm{BuOK}$, THF, $20^{\circ} \mathrm{C}, 12 \mathrm{~h}$; c: $\mathrm{KOH}, \mathrm{HOCH}_{2} \mathrm{CH}_{2} \mathrm{OH}, 180^{\circ} \mathrm{C}, 30 \mathrm{~min}$ for $\mathbf{1 0 a}, \mathrm{CF}_{3} \mathrm{COOH}-\mathrm{CH}_{2} \mathrm{Cl}_{2}, 20^{\circ} \mathrm{C}, 1 \mathrm{~h}$ for $\mathbf{1 0 b}$.

An interesting feature of this synthesis is the use of allylic sulfone in place of vinylic sulfone. The Hopkins-Fuchs procedure in this case leads to the product with shifted double bond, in exact accordance with the stereochemistry of elimination.

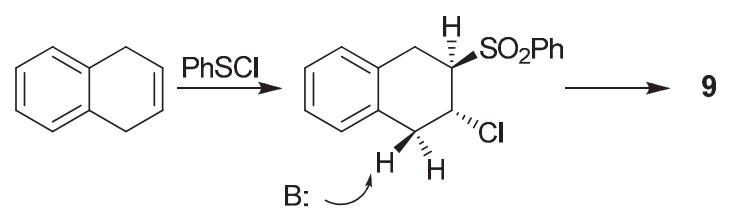

This result might be fatal for the following transformations, as Barton-Zard reaction requires vinylic sulfones because the key stage of this reaction is Michael-type addition to the activated double bond. Nevertheless, as we have established allylic sulfones can be used in the Barton-Zard reaction as well if a slight excess of strong base is introduced to the reaction. This finding can be accounted for by prototropic rearrangement of these sulfones, with allylic sulfone being more stable due to conjugation of double bond and aromatic ring, and vinylic sulfone being the reactive species.

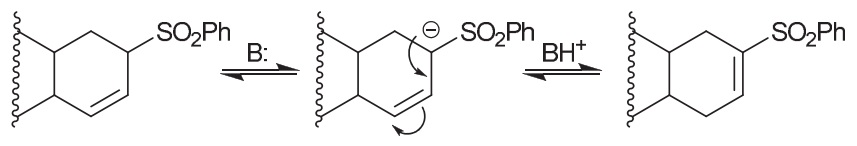

The discovered modification is of prime importance for the realization of synthetic strategy. The elimination from cyclic chlorosulfenylation adducts often gives allylic sulfones, which otherwise should not be useful. Thus, the allylsulfonylic modification of the Barton-Zard reaction can be used in the synthesis of pyrroles. Recently, we have used this modification in the synthesis of tetraanthraporphyrins ${ }^{[19]}$ using a similar approach.

Dihydrobenzoisoindole 8 was used without further purification in the Lindsey's condensation with aromatic aldehydes. Interestingly, in this case the aromatization of porphyrinogene was accompanied by further aromatization of annelated rings. The isolation of intermediate porphyrins, similarly to that used in the synthesis of tetrabenzoporphyrins, could not be achieved. Therefore, we introduced an additional amount of DDQ during the quenching of porphyrinogene and allowed for longer reaction time, and directly obtained tetraaryltetranaphthoporphyrins in good yields and high purity achieved after filtration through silica layer and recrystallization.

The solubility of the obtained porphyrins is high enough to allow for taking their NMR spectra in common solvents at room temperature. However, free bases of these porphyrins are not useful in this respect due to heavy broadening of resonance lines, likely because of fluxional behavior due to both tautomeric shift of inner protons, and non-planar macrocycle inversion, similarly to what is observed in tetraaryltetrabenzoporphyrins. The resolution can be improved by registering the spectra in pyridine $\left[\mathrm{D}_{5}\right]$ at elevated temperature. However, it is more convenient and consistent to register well-resolved spectra of the diprotonated forms, e.g. bis-trifluoroacetate salts. In order to obtain a high resolution for all resonance lines, these salts must be thoroughly purified from traces of free acid by recrystallization or high-vacuum drying. The resonance lines of mesoaryl protons are shifted downfield to $8.2-8.6 \mathrm{ppm}$, what is likely due to the deshielding effect of naphthalene fragments ring current. Conversely, the meso-aryl groups induce an upfield shift of protons of inner rings of naphthalene residues. This effect is also likely the reason of unusual downfield shift of the internal NH-protons. In the majority of porphyrins (including tetrabenzoporphyrins) and their dications the protons' resonances are located in the negative part of chemical shifts scale between -1 and -4 ppm, while for tetraaryltetrabenzoporphyrins there are no resonance lines below 0 ppm. Characteristic broadened resonance lines can be found instead between $+2-+3 \mathrm{ppm}$. Such a strong downfield shift can be tentatively attributed to the deshielding effect of superposition of ring currents of aromatic naphthalene subsystems. Similar effects can be traced in ${ }^{13} \mathrm{C}$ NMR spectra.

The electronic absorption spectra of the free bases show very intense $Q$-bands between 700 and $740 \mathrm{~nm}$. The position of absorption bands depend only weakly on the nature of meso-aryl substituents, which is a common trend in porphyrin chemistry, as the conjugation between meso-aryl substituents and porphyrin core is generally very weak. The comparison between the spectra of porphyrins containing $p$-substituted aryls and 3,5-disubstituted aryls reveal an interesting trendwhile the position of main maxima are practically the same, the shape of lines is dramatically different. For example, the most characteristic among the second group porphyrins 
the compound $12 \mathrm{~g}$, containing the bulky tert-butyl groups in 3,5-positions of meso-aryls, displays the well-resolved absorption bands, while porphyrins of the first group show only a rough envelope of the fine structure, which can only be guessed from hardly discernible shoulders or inflections (Figure 1). Though the reasons of such difference require special investigation, which will be reported elsewhere, here we note that the most probable cause of this phenomenon is the suppression of aggregation in the case when meso-aryls bear 3,5-substituents which are situated at both sides of the macrocycle and may interfere with stacking intramolecular interactions.

The dihydroisoindole approach was used also for the synthesis of so far unknown 5,15-diaryltetranaphthoporphyrin. The interest to this molecule was spawned by our study of the structureproperties relationships in the tetrabenzoporphyrin series. We have shown that one or two meso-aryl groups do not effect out-of-plane deformation of porphyrin core, and sterical strain induced by meso-aryls interaction with benzorings is relieved by in-plane distortion by elongation of the core ${ }^{[1]}$ Due to the planarity of the core, such porphyrins exhibit outstanding optical properties very similar to those of meso-unsubstituted tetrabenzoporphyrin, while at the same time meso-aryl rings furnish additional degrees of freedom allowing for attachment of functional groups, etc. We expected the same trends in the tetranaphthoporphyrin series, where this possibility might be even more welcome.

In planning the synthesis we took into consideration that the porphyrin to be synthesized should be more prone to aggregation than the saddle distorted tetraaryltetranaphthoporphyrins, and chose 3,5-di-tertbutylphenyl groups to restrict aggregation and improve solubility. The synthesis involved the preparation of dipyrromethane $\mathbf{1 3}$ by condensation of ethoxycarbonyl derivative of dihydrobenzoisoindole 10a with dimethoxymethane. Dipyrromethane $\mathbf{1 3}$ was condensed

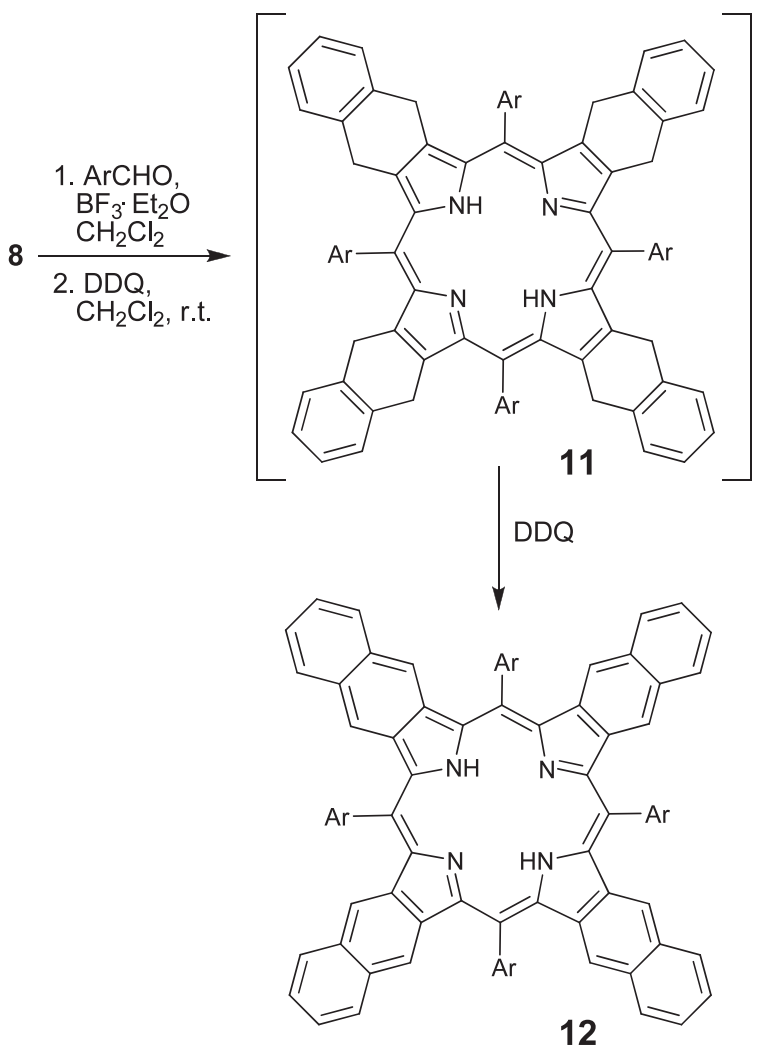

$\mathrm{Ar}=\mathrm{H}(12 \mathrm{a}), p-\mathrm{COOMe}(12 \mathrm{~b}), \mathrm{NO}_{2}(12 \mathrm{c})$, OMe (12d), $\mathrm{Br}(12 \mathrm{e}), 3,5-(\mathrm{OMe})_{2}(12 \mathrm{f})$, $(\mathrm{tBu})_{2}(12 \mathrm{~g}),(\mathrm{COOMe})_{2}(12 \mathrm{~h})$

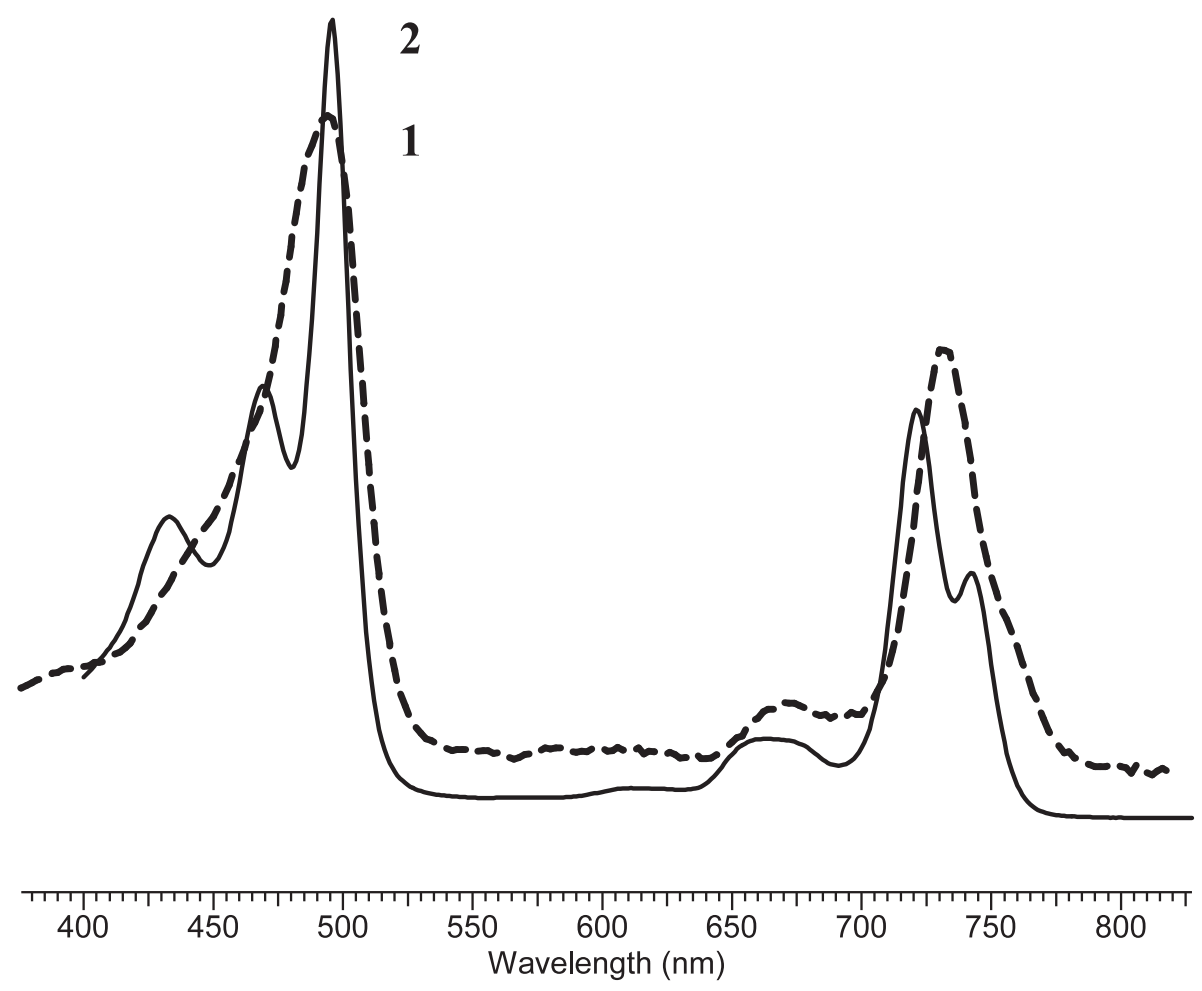

Figure 1. The absorption spectra of tetraaryltetranaphthoporphyrins 12a (dashed line) and 12g (solid line). 


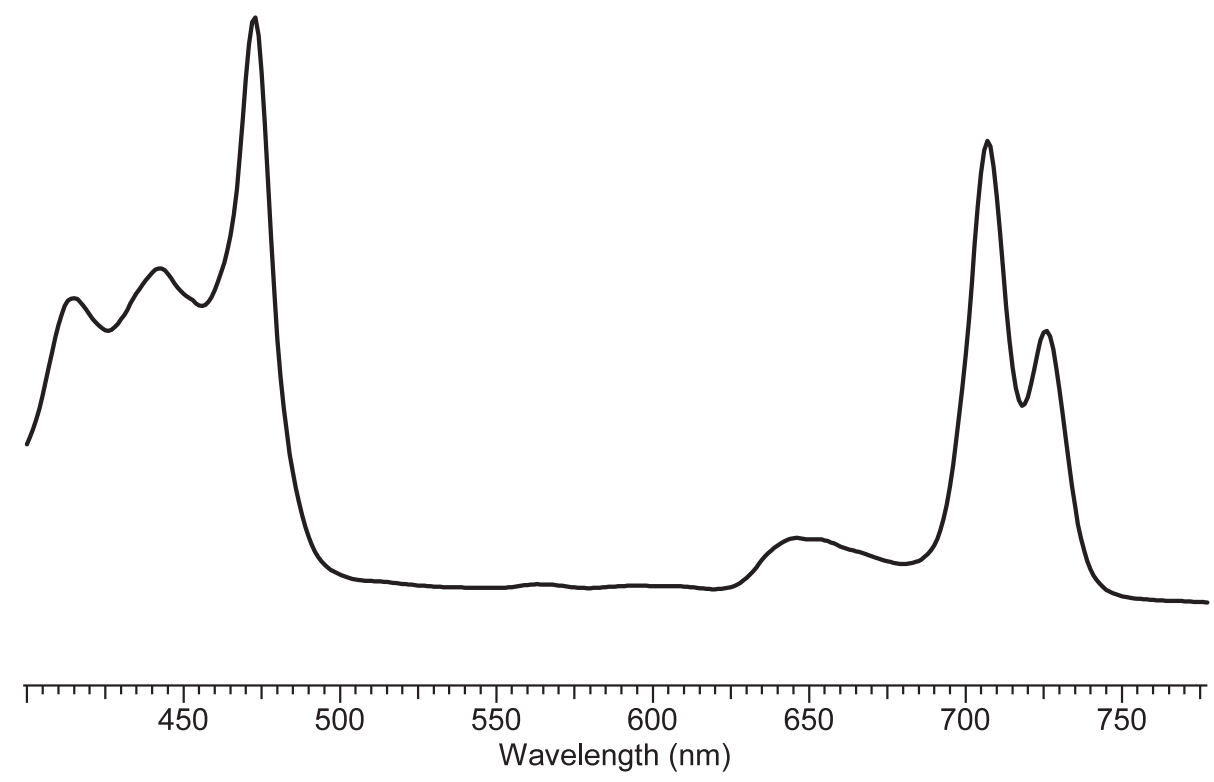

Figure 2. The electronic absorption spectrum of free base porphyrin 14 in $\mathrm{CH}_{2} \mathrm{Cl}_{2}$.

with the respective benzaldehyde, and the reaction mixture was quenched with DDQ to afford directly the expected diarylporphyrin $\mathbf{1 4}$ in a good yield.

Even in spite of the presence of bulky meso-aryl substituents porphyrin $\mathbf{1 4}$ is a sparingly soluble material. Nevetheless, its solubility is sufficient for efficient purification by recrystallization, which enables to obtain this porphyrin in an analytically pure form. In NMR spectra of the dication form the signals of naphthalene protons are broadened, while the signals of meso-aryls are well resolved. Porphyrin 14 was characterized by MALDITOF mass-spectrometry and elemental analysis, as well as electronic absorption spectroscopy. The absorption spectra (Figure 2) of porphyrin 14 resemble those of tetraaryltetranaphthoporphyrins, with a notable difference. Both Soret and $Q$-bands exhibit characteristic splitting. This feature is reminiscent of a similar phenomenon, which we have noted in the spectra of 5,15-diaryltetrabenzoporphyrins, and interpreted as a characteristic token of a planar structure of macroheterocycle ("planarity signature"). Therefore, we believe that similarly substituted tetranaphthoporphyrins, such as $\mathbf{1 4}$ may be also planar. The detailed investigation of structural and photophysical trends in tetranaphthoporphyrin series will be reported separately.

\section{Conclusions}

Thus, we have developed a new versatile approach to linearly annelated extended porphyrins via the closest possible precursors of unstable $2 \mathrm{H}$-isindoles - 4,7-dihydroisoindole and its benzoannelated derivative. These precursors can be obtained in high yields starting from commercially available simple starting materials, and used for the preparation of various tetrabenzo- and tetranaphthoporphyrins using the well-established Lindsey's porphyrin synthesis procedures. The aromatization of annelated rings by DDQ is performed under very mild conditions, either spontaneously during the quenching of porphyrinogenes after Lindsey's
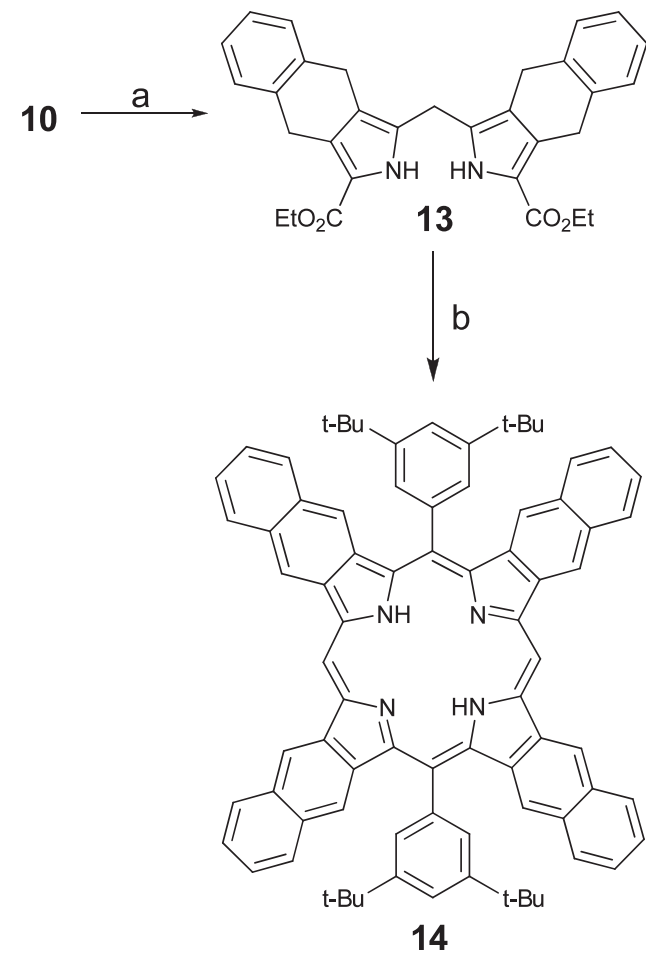

Scheme 4. a: $\mathrm{CH}_{2}(\mathrm{OMe})_{2}$, TsOH, $\mathrm{CH}_{3} \mathrm{COOH}, 20^{\circ} \mathrm{C}, 12 \mathrm{~h}$; b: 1 . $\mathrm{KOH}, \mathrm{HOCH}_{2} \mathrm{CH}_{2} \mathrm{OH}, 180^{\circ} \mathrm{C}, 30 \mathrm{~min} ; 2$. 3,5- ${ }^{t} \mathrm{Bu}_{2} \mathrm{C}_{6} \mathrm{H}_{5} \mathrm{CHO}$, $\mathrm{CF}_{3} \mathrm{COOH}, \mathrm{CH}_{2} \mathrm{Cl}_{2}, 6 \mathrm{~h} ; 2$. DDQ, $12 \mathrm{~h}$.

condensation, or by short treatment of intermediate partially hydrogenated porphyrins. The method is equally applicable to tetraarylsubstituted porphyrins, and to partially mesosubstituted porphyrins, which are of special interest because of planarity of porphyrin core.

Acknowledgements. The Russian Foundation of Basic Research (grant RFBR 07-03-01121) is gratefully acknowledged for financial support. 


\section{References}

1. Lash T.D. Syntheses of Novel Porphyrinoid Chromophores. In: The Porphyrin Handbook (Kadish K.M., Smith K.M., Guilard R., Eds) New York: Academic Press, 2000, Vol. 2, 125-200.

2. Guha S., Kang K., Porter P., Roach J. F., Remy D. E., Aranda F.J., Rao D.V.G.L.N. Optics Lett. 1992, 17, 264-266; Brunel M., Chaput F., Vinogradov S.A., Campagne B., Canva M., Boilot J.P., Brun A. Chem. Phys. 1997, 218, 301-307; Shea P.B., Chen C., Kanicki J., Pattison L.R., Petroff P., Yamada H., Ono N. Appl. Phys. Lett. 2007, 90, 233107; Borek C., Hanson K., Djurovich P.I., Thompson M.E., Aznavour K., Bau R., Sun Y.R., Forrest S.R., Brooks J., Michalski L., Brown J. Angew. Chem., Int. Ed. 2007, 46, 1109-1112; Sun Y., Borek C., Hanson K., Djurovich P.I., Thompson M.E., Brooks J., Brown J.J., Forrest S.R. Appl. Phys. Lett. 2007, 90, 213503; Kobayashi N., Nevin W.A., Mizunuma S., Awaji H., Yamaguchi M. Chem. Phys. Lett. 1993, 205, 51-54.

3. Seidel K.F., Koehler M. Phys. Rev. B 2008, 78, 235308; Aramaki S., Sakai Y., Ono N. Appl. Phys. Lett. 2004, 84, 2085 2087; Xu M. S., Ohno A., Aramaki S., Kudo K., Nakamura M. Org. Electronics 2008, 9, 439-444.

4. Kikuchi E., Kitada S., Ohno A., Aramaki S., Maenosono S. Appl. Phys. Lett. 2008, 92, 173307; Yamada H., Kamio N., Ohishi A., Kawano M., Okujima T., Ono N. J. Porphyrins Phthalocyanines 2007, 11, 383-389; Perez M.D., Borek C., Djurovich P.I., Mayo E.I., Lunt R.R., Forrest S.R., Thompson M.E. Adv. Mater. 2009, 21, 1517-1520.

5. Vinogradov S.A., Wilson D.F. J. Chem. Soc. Perkin Transactions 1995, 2, 103-111; Dunphy I., Vinogradov S.A., Wilson D.F. Anal. Biochem. 2002, 310, 191-198; Finikova O., Galkin A., Rozhkov V., Cordero M., Hagerhall C., Vinogradov S. J. Am. Chem. Soc. 2003, 125, 4882-4893; Rietveld I. B., Kim E., Vinogradov S.A. Tetrahedron 2003, 59, 3821-3831; Apreleva S.V., Wilson D.F., Vinogradov S.A. Appl. Optics 2006, 45, 8547-8559; Wilson D.F., Lee W.M.F., Makonnen S., Finikova O., Apreleva S., Vinogradov S.A. J. Appl. Physiol. 2006, 101, 1648-1656; Finikova O. S., Troxler T., Senes A., DeGrado W.F., Hochstrasser R.M., Vinogradov S.A. J. Phys. Chem. A 2007, 111, 6977-6990; Tao Z.M., Jones E., Goodisman J., Souid A.K. Anal. Biochem. 2008, 381, 43-52; Tao Z., Goodisman J., Souid A.K. J. Phys. Chem. A 2008, 112, 1511-1518; Tao Z.M., Ahmad S.S., Penefsky H.S., Goodisman J., Souid A.K., Mol. Pharm. 2006, 3, 762-772; Lebedev A., Cheprakov A., Sakadzic S., Boas D., Wilson D., Vinogradov S. ACS Appl. Mater. Interfaces 2009, 1, 1292-1304; Borisov M., Nuss G., Haas W., Saf R., Schmuck M., Klimant I. J. Photochem. Photobiol. A 2009, 201, 128-135.

6. Friedberg J.S., Skema C., Baum E.D., Burdick J., Vinogradov S.A., Wilson D.F., Horan A.D., Nachamkin I. J. Antimicrobial Chemotherapy 2001, 48, 105-107; Ongayi O., Gottumukkala V., Fronczek F.R., Vicente M.G.H. Bioorg. Med. Chem. Lett. 2005, 15, 1665-1668; Gottumukkala V., Ongayi O., Baker D.G., Lomax L.G., Vicente M.G.H. Bioorg. Med. Chem. 2006, 14, 1871-1879.
7. Sommer J.R., Farley R.T., Graham K.R., Yang Y., Reynolds J.R., Xue J., Schanze K.S. ACS Appl. Mater. Interfaces 2009, $1,274-278$.

8. Baluschev S., Yakutkin V., Miteva T., Avlasevich Y., Chernov S., Aleshchenkov S., Nelles G., Cheprakov A., Yasuda A., Mullen K., Wegner G. Angew. Chem., Int. Ed. 2007, 46, 76937696; Baluschev S., Yakutkin V., Wegner G., Miteva T., Nelles G., Yasuda A., Chernov S., Aleshchenkov S., Cheprakov A. Appl. Phys. Lett. 2007, 90, 181103; Baluschev S., Yakutkin V., Miteva T., Wegner G., Roberts T., Nelles G., Yasuda A., Chernov S., Aleshchenkov S., Cheprakov A. New J. Phys. 2008, 10, 1-12; Singh-Rachford T.N., Castellano F.N. Inorg. Chem. 2009, 48, 2541-2548; Singh-Rachford T.N., Haefele A., Ziessel R., Castellano F.N. J. Am. Chem. Soc. 2008, 130, 16164.

9. Miteva T., Yakutkin V., Nelles G., Baluschev S. New J. Phys. 2008, 10, 103002 .

10. Ekins-Daukes N.J., Schmidt T.W. Appl. Phys. Lett. 2008, 93, 063507.

11. Barret P.A., Linstead R.P., Rundall F.G., Tuey G.A.P. J. Chem. Soc. 1940, 1079-1092.

12. Kopranenkov V.N., Makarova E.A., Luk'yanets E.A. $Z h$ Obshch. Khim. 1981, 51, 2727-2730.

13. Kopranenkov V.N., Makarova E.A., Dashkevich S.N., Luk'yanets E.A. Khim. Geterotsikl. Soed. 1988, 773-779.

14. Ito S., Murashima T., Uno H., Ono N. Chem. Commun. 1998, 1661-1662.

15. Ito S., Ochi N., Uno H., Murashima T., Ono N. Chem. Commun. 2000, 893-894.

16. Lash T.D. Energy Fuels 1993, 7, 166-171.

17. Finikova O.S., Cheprakov A.V., Beletskaya I.P., Carroll P.J., Vinogradov S.A. J. Org. Chem. 2004, 69, 522-535.

18. Finikova O.S., Aleshchenkov S.E., Brinas R.P., Cheprakov A.V., Carroll P.J., Vinogradov S.A. J. Org. Chem. 2005, 70, 4617-4628

19. Yakutkin V., Aleshchenkov S., Chernov S., Miteva T., Nelles G., Cheprakov A., Baluschev S. Chem.-Eur. J. 2008, 14, 98469850 .

20. Filatov M.A., Lebedev A.Y., Vinogradov S.A., Cheprakov A.V. J. Org. Chem. 2008, 73, 4175-4185

21. Cheprakov A.V., Filatov M.A. J. Porphyrins Phthalocyanines 2009, 13, 291-303.

22. Fuhrhop J.H., Hosseinpour D. J. Liebigs Ann. Chem. 1985, 689-695.

23. Filatov M.A., Cheprakov A.V., Beletskaya I.P. Eur. J. Org. Chem. 2007, 3468-3475

24. Otten A., Namyslo J.C., Stoermer M., Kaufmann D.E. Eur. J. Org. Chem. 1998, 1997-2001.

25. Barton D.H.R., Kervagoret J., Zard S.Z. Tetrahedron 1990, 46, 7587-7598.

26. Filatov M.A., Cheprakov A. V. Org. Biomol. Chem. submitted for publication

27. Hueckel W., Schlee R. Chem. Ber. 1955, 88, 346.

28. Hopkins P.B., Fuchs P.L. J. Org. Chem. 1978, 43, 1208-1217.

29. Lebedev A.Y., Filatov M.A., Cheprakov A.V., Vinogradov S.A. J. Phys. Chem. A 2008, 112, 7723-7733. 